\title{
Effect of TGF- $\beta 1$ on Water Retention Properties of Healthy and Osteoarthritic Chondrocytes
}

\author{
Tehmeena Israr Raja, Seyed Ali Khaghani, Muhammad Sohail Zafar, Zohaib \\ Khurshid, Masoud Mozafari, Mansour Youseffi, Farshid Sefat
}

\begin{abstract}
Articular cartilage, a connective tissue, contains chondrocytes and glycosaminoglycans (GAGs) which aid in water retention, providing the tissue with its magnificent ability to prevent friction, withstand loads and absorb compressive shocks however, cartilage, does not have the ability to regenerate and repair. Osteoarthritis (OA) is a progressive degenerative disease, which includes reduction of cartilage thickness between two bones in a joint, causing painful bone-to-bone contact. OA affects over 8 million people in the UK alone., and as the primary causes are unknown, available treatments including surgical and non-surgical techniques which only reduce the symptoms created by the disorder instead of providing a cure. This project focused on utilizing TGF- $\beta 1$, a cytokine found in elevated amounts in healthy cartilage when compared to degraded cartilage, in order to observe the effects of the growth factor on both healthy and osteoarthritic chondrocytes. The healthy and the osteoarthritic chondrocytes were cultured in two different media (DMEM with and without TGF- $\beta 1$ ) before utilizing the SpectraMax M2/M2e plate reader to observe and analyze the effect of TGF- $\beta 1$ on water retention properties of cells. This has been achieved by quantifying the GAG content using DMMB dye. Results showed that although TGF- $\beta 1$ did displayed an increase in glycosaminoglycan synthesis, the statistical increase was not vast enough for the alternative hypothesis to be accepted; further experimentation with TGF- $\beta 1$, alongside other cytokines within the growth factor family is needed to perceive the true influence of the growth factor on un cured degenerative diseases. It was concluded that both the healthy and osteoarthritic cells treated with TGF- $\beta 1$ absorbed considerably more DMMB in comparison to the cells, suggesting that TGF- $\beta 1$ indeed works to aid in water retention. TGF- $\beta 1$ is a key factor to be exploited when constructing treatments for osteoarthritis.
\end{abstract}

Keywords: TGF- $\beta 1$; cytokines; water retention; articular cartilage; chondrocytes; osteoarthritis; immunocytochemistry; DMMB. 


\section{Introduction}

Osteoarthritis is a progressive degenerative disease, which includes reduction of cartilage thickness between two bones in a joint, causing painful bone-to-bone contact. Cartilage is retained within the synovial capsule present at the sight of each joint, reducing friction and absorbing shock; the synovium which surrounds each capsule when one is suffering from OA swells, and as the synovium becomes inflamed, it produces a diseased synovial fluid which further affects the joint. This fluid remains in contact with the ligaments, causing them to contract leading to a reduction in thickness; 
the muscle attached to the ligament aiding in the affected joints movement is weakened as a result, and said muscle becpmesiatiffaraducimorange of motion. In osteoarthritis, proteoglycans from articular cartilage are said to be lost [1]. There are many factors which promote arthritis but one of the main hallmarks of OA occurring is a reduction in

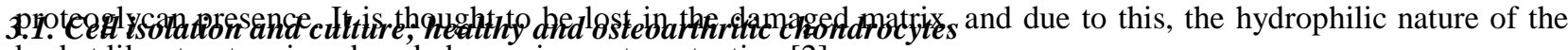
basket like structure is reduced, decreasing water retention [2].

Articular cartilage is a highly specialised form of tissue. Being avascular, it can only gain its nutrients through diffusion. The extracellular matrix consists of mainly two macromolecules including the proteoglycan aggrecan and also collagen (mostly type II). Chondrocytes, the main resident cell in articular cartilage coordinate the synthesis, maintenance and degradation of proteins existent in the extracellular matrix. In cartilage that is considered healthy, the 
chondrocytes are in a state of dormancy due to the balance of synthetic and catabolic actions whereas when a cartilage has been partially degraded or when trauma has occurred to the joint, water retention properties are lost and an up regulation of catabolic pathways occur [2]. Damage to the cartilage that occurs in osteoarthritic situations may be a result of a misbalance between factors, with catabolic overpowering anabolic reactions.

Despite many attempts, a method of repairing cartilage that is damaged when osteoarthritis occurred had not been finalised. An existing approach considers fluctuating metabolic imbalances back to the original state by causing a stimulation acting upon the anabolic reactions. TGF- $\beta 1$ is an anabolic factor which is directly linked to maintaining the cartilage, working by stimulating ECM production, downgrades degrading cytokines [3] and therefore, is an 
appropriate option to be observed; TGF- $\beta 1$ signalling serves as a potential key target for therapeutic intervention for 3h Mrtatinastand

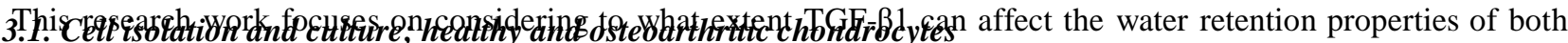
healthy and osteoarthritic chondrocytes taken from the knee joint of a Swiss white mouse. By manipulating the media in which the cells are cultured (using the growth factor as a variable) and through the staining of proteoglycans, quantification of GAGS can be achieved/ and absorbance of stain can be measured. If the TGF- $\beta 1$ demonstrates significant differences in terms of increasing water retention (increased absorbance in the samples containing the growth factor in comparison to the samples cultured in ordinary media), it can be confirmed that TGF- $\beta 1$ is a focal point for curing the degenerative disease [7]. 


\section{Aims and Objectives}

The aim of this research work was to investigate the effect of TGF- $\beta 1$ on water retention properties of glycosaminoglycans on both healthy and osteoarthritic chondrocytes. 


\section{Materials and Methods}

\subsection{Cell isolation and culture; healthy and osteoarthritic chondrocytes}

Chondrocyte were isolated and purified from the knee joint of a Swiss white Mouse. Reagents were used included high glucose DMEM $(4500 \mathrm{mg} / \mathrm{l}$ glucose) inclusive of serum, Hanks balanced salt solution (HBSS), trypsin and collagenase type-IA $10 \% \mathrm{v} / \mathrm{v}$ [8]. The DMEM was induced with $20 \% \mathrm{v} / \mathrm{v}$ fetal bovine serum (FBS), $2.5 \mathrm{U} / \mathrm{ml} \mathrm{L-}$ glutamine, $100 \mathrm{U} / \mathrm{ml}$ penicillin, $0.1 \mathrm{mg} / \mathrm{ml}$ streptomycin and $1 \mu \mathrm{g}$ Amphotericin B [9]. Cartilage samples were carefully isolated from 7 days old euthanized Swiss white mice at the knee joint as described previously [10]. Briefly, the tissue 
was washed with HBSS, eliminating the chances of having tissue other than cartilaginous (such as adipose or blood cells) present in the culture sample. The cartilage samples were soaked in $4 \mathrm{ml}$ of trypsin at 37 degrees for 15 minutes and stirred using magnetic stirrer. The supernatant was transferred into a sterilised $15 \mathrm{ml}$ centrifuge tube containing $6 \mathrm{ml}$ DMEM and 10\%FBS in order to stop activation of trypsin. After three repeats, these suspensions were combined and centrifuged for 5 minutes at 2000rpm. The excess media was discarded before the pellet was re-suspended in $1 \mathrm{ml}$ collagenase, stirred for 90 minutes at 37 degrees. The suspension was combined with $4 \mathrm{ml}$ of DMEM in order to 


\section{Materials and Methods}

\subsection{Cell isolation and culture; healthy and osteoarthritic chondrocytes}

prevent further action of collagenase, and centrifuged for 5 minutes at 2000rpm before the excess media was discarded. The pellet was further re-suspended in $6 \mathrm{ml}$ of DMEM induced with $0.1 \mathrm{mg} / \mathrm{ml}$ hyaluronic acid before being placed in a $25 \mathrm{~cm}^{2}$-culture flask and incubated at 37 degrees for 24 hours [8].

\subsubsection{Primary cell culture}


A sample of the isolated chondrocytes were taken and chemically induced in order to replicate osteoarthritic conditions; the two groups included healthy and osteoarthritic chondrocytes. Both variations of cells were cultured and reagents included; hanks balanced salt solution (HBSS), trypsin, and High glucose DMEM (4500mg/l) induced with $10 \%$ fetal bovine serum (FBS), $2.5 \mathrm{U} / \mathrm{ml} \mathrm{L-glutamine,} 100 \mathrm{U} / \mathrm{ml}$ penicillin, $0.1 \mathrm{mg} / \mathrm{ml}$ streptomycin and $1 \mu \mathrm{g}$ Amphotericin B $[8,10]$. The isolated chondrocyte cell culture flask was taken and the media was removed, leaving the monolayer of chondrocytes. The chondrocyte monolayer of cells was washed with $3 \mathrm{ml}$ of HBSS after the media 


\section{Materials and Methods}

\subsection{Cell isolation and culture; healthy and osteoarthritic chondrocytes}

was removed in order to deactivate the serum present in the DMEM. After three repeats, $6 \mathrm{ml}$ of trypsin was added to the culture flask and incubated at 37 degrees for 2 minutes. After $90 \%$ of cells were dislodged, $12 \mathrm{ml}$ of the DMEM was added to the cell suspension. The cell suspension was then split into two sterilised $9 \mathrm{ml}$ labelled centrifuge tubes, and centrifuged at 1700rpm for 5 minutes. The supernatant of tube 1 was removed, and the pellet re-suspended in $6 \mathrm{ml}$ DMEM, transferred into a $25 \mathrm{~cm}^{2}$ cell culture flask labelled $\mathrm{HCH}$ (for healthy chondrocytes) and incubated at 37 degrees for 24 hours. The supernatant from tube 2 was removed, the pellet re-suspended in 6ml DMEM containing 
chemicals that induce osteoarthritic conditions, transferred into a second (labelled $\mathrm{OACH}$ ) $25 \mathrm{~cm}^{2}$-culture flask and also incubated at 37 degrees for 24 hours. After 24 hours, samples of healthy chondrocytes and osteoarthritic chondrocytes had been obtained; both cell types underwent a sub culture; media from both culture flasks (HCH and $\mathrm{OACH}$ ) was removed, the monolayers rinsed with $1 \mathrm{ml}$ HBSS twice, $2 \mathrm{ml}$ trypsin added to both flasks before incubation at 37 degrees for 2 minutes, furthermore combined with $6 \mathrm{ml}$ of DMEM. The cell suspensions were centrifuged for 5 minutes at 1700rpm before the pellets were re-suspended in High glucose DMEM (4500mg/l) induced with $10 \%$ fetal 


\section{Materials and Methods}

\subsection{Cell isolation and culture; healthy and osteoarthritic chondrocytes}

bovine serum (FBS), $2.5 \mathrm{U} / \mathrm{ml} \mathrm{L-glutamine,} 100 \mathrm{U} / \mathrm{ml}$ penicillin, $0.1 \mathrm{mg} / \mathrm{ml}$ streptomycin and $1 \mu \mathrm{g}$ Amphotericin B. $6 \mathrm{ml}$ of healthy chondrocyte suspension were again transferred into a sterilised $25 \mathrm{~cm}^{2}$ labelled culture flask $(\mathrm{HCH}+$ $48 \mathrm{hr}$ ) using a pipette, and likewise $6 \mathrm{ml}$ of the osteoarthritic chondrocyte suspension was transferred into a $25 \mathrm{~cm}^{2}$ labelled culture flask $(\mathrm{OACH}+48 \mathrm{hr})$; both samples incubated for a further 24 hours at 37 degrees.

\subsubsection{Secondary culture and TGF-ק1 treatment}


Healthy and osteoarthritic chondrocytes were further cultured with two different types of media. Media type I included high glucose DMEM (4500mg/l) supplemented with 10\% fetal bovine serum (FBS), $2.5 \mathrm{U} / \mathrm{ml}$ L-glutamine, $100 \mathrm{U} / \mathrm{ml}$ penicillin, $0.1 \mathrm{mg} / \mathrm{ml}$ streptomycin and $1 \mu \mathrm{g}$ Amphotericin B. Media type II included high glucose DMEM (4500mg/l) supplemented with $10 \%$ fetal bovine serum(FBS), $2.5 \mathrm{U} / \mathrm{ml}$ L-glutamine, $100 \mathrm{U} / \mathrm{ml}$ penicillin, $0.1 \mathrm{mg} / \mathrm{ml}$ streptomycin 


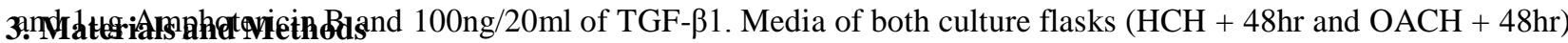
was removed; the monolayers rinsed with $1 \mathrm{ml}$ HBSS twice, $2 \mathrm{ml}$ trypsin added to both flasks before incubation at 37

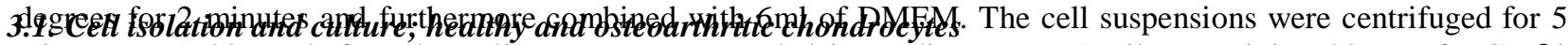
minutes at $1700 \mathrm{rpm}$ before the pellets were re-suspended in media type I. A vile containing $20 \mu \mathrm{m}$ of TGF- $\beta 1$ $(100 \mathrm{ng} / 20 \mathrm{ml})$ was centrifuged before extraction, combined with $5 \mathrm{ml}$ of DMEM (media type I) in order to create media type II $(20 \mathrm{ng} / \mathrm{ml}) .300 \mu 1$ of healthy cell suspension was placed into a labelled petri dish with an additional $300 \mu 1$ of media type I. $300 \mu \mathrm{l}$ of healthy cell suspension was placed into a second labelled petri dish (healthy chondrocyte + TGF- $\beta 1$ ) with an additional $300 \mu 1$ of media type II. $300 \mu 1$ of osteoarthritic cell suspension was placed into a third labelled petri dish (osteoarthritic chondrocyte control) with an additional $300 \mu$ l of media type I. $300 \mu$ l of osteoarthritic 
cell suspension was placed into a fourth labelled petri dish (osteoarthritic chondrocyte + TGF- $\beta 1$ ) with an additional $300 \mu 1$ of media type II.

\subsection{Analysis of glycosaminoglycan content using the SpectraMax M2/M2 ${ }^{e}$ plate reader}

A plate reader (SpectraMax M2/M2e ${ }^{\mathrm{e}}$, USA) was used to analyse the 4 culture samples described previously for GAG concentration with absorbance read at $525 \mathrm{~nm}$. The plate reader was calibrated to allow 5-second shake before the first read and left to analyse the well plate. Reagents included DMMB dye and were prepared from 0.00064g of pure $\mathrm{DMMB}$, induced in $400 \mathrm{ml}$ deionised water, $1.216 \mathrm{~g}$ glycine, $0.64 \mathrm{~g}$ sodium chloride and $38 \mathrm{ml}$ of $0.1 \mathrm{M}$ acetic acid [11]. 
Cell suspension was taken from the four petri dishes and centrifuged separately at 800rmp for 5 minutes. A sterile 96

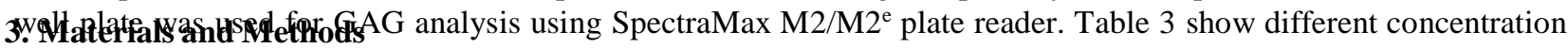
of DMMB for both osteoarthritic and healthy cells.

\subsection{Cell isolation and culture; healthy and osteoarthritic chondrocytes}

Table 1. Different concentration of reagents and DMMB for analysis of GAGs.

\begin{tabular}{|l|l|}
\hline \multicolumn{2}{|c|}{ Osteoarthritis Cells } \\
\hline $90 \mu 1$ of osteoarthritic cell supernatant induced with TGF- $\beta 1$ & $190 \mu 1$ DMMB \\
\hline $60 \mu 1$ of degraded cell supernatant induced with TGF- $\beta 1$ & $220 \mu 1$ DMMB \\
\hline $30 \mu 1$ of degraded cell supernatant induced with TGF- $\beta 1$ & $250 \mu 1$ DMMB \\
\hline
\end{tabular}




\begin{tabular}{|l|l|}
\hline $90 \mu 1$ of OA cell control supernatant & $190 \mu 1$ DMMB \\
\hline $60 \mu 1$ of OA cell control supernatant & $220 \mu 1$ DMMB \\
\hline $30 \mu 1$ of OA cell control & $250 \mu 1$ DMMB \\
\hline \multicolumn{2}{|c|}{ Healthy Cells } \\
\hline $90 \mu 1$ of healthy cell supernatant control & $190 \mu 1$ DMMB \\
\hline $60 \mu 1$ of healthy cell supernatant control & $220 \mu 1$ DMMB \\
\hline $30 \mu 1$ healthy cell supernatant control & $250 \mu 1$ DMMB \\
\hline $90 u 1$ of healthy cell supernatant induced with TGF- $\beta 1$ & $190 \mu 1$ DMMB \\
\hline $60 \mu 1$ of healthy cell supernatant induced with TGF- $\beta 1$ & $220 \mu 1$ DMMB \\
\hline
\end{tabular}




\section{Materials and Methods}

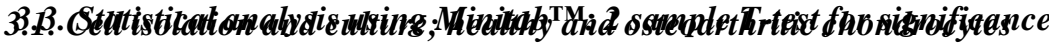

Minitab, an analysis software, was utilised in order to easily assimilate statistical data. 2-sample T test was used for this research work to quantify the difference present in glycosaminoglycan synthesis. 



\section{Result and Discussion}

\subsection{Cell isolation and culture; healthy and osteoarthritic chondrocytes}

\section{Table 2 - Cell Isolation}

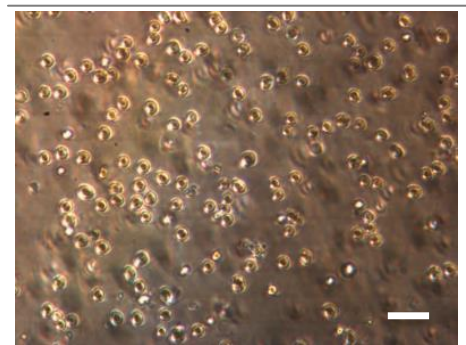

Shows the original primary cells floating after isolation, dispersed in fresh media before culturing to double. These cells were considered healthy, and demonstrated a rounded shape as chondrocytes show in vitro. The cells were then incubated for attachment and division to occur.

Figure 1 - Isolated healthy chondrocytes, $($ Scale bar $=100)$.

Primary cell culture

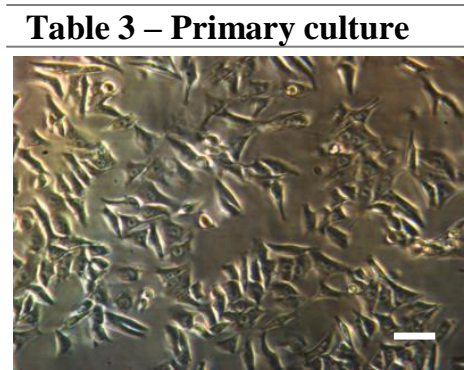

Figure 2 - Healthy chondrocytes cultured after 24 hours, (Scale bar $=100)$.

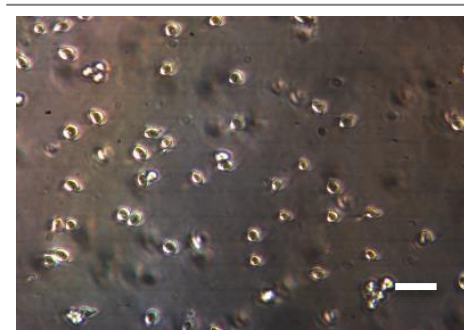

Shows healthy chondrocytes in DMEM after being dislodged with trypsin and re-suspended [24 hours of culturing].

Figure 3 - Trypsinisation of healthy chondrocytes after 24 hours of culture, (Scale bar $=100)$.

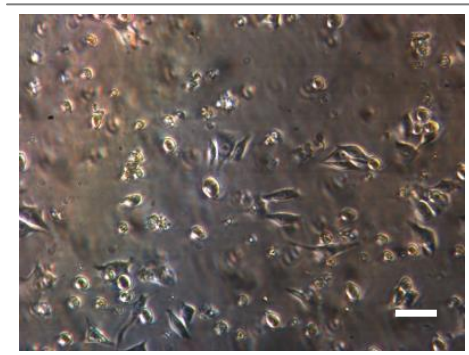

Shows degraded chondrocyte cells in DMEM (Created to replicate osteoarthritic conditions). These cells have been detached and re-suspended in high glucose DMEM 24 hours after first culture.

Figure 4 - Trypsinsation and re-suspension of Osteoarthritic chondrocytes 24 hours after culture, $($ Scale bar $=100)$. 


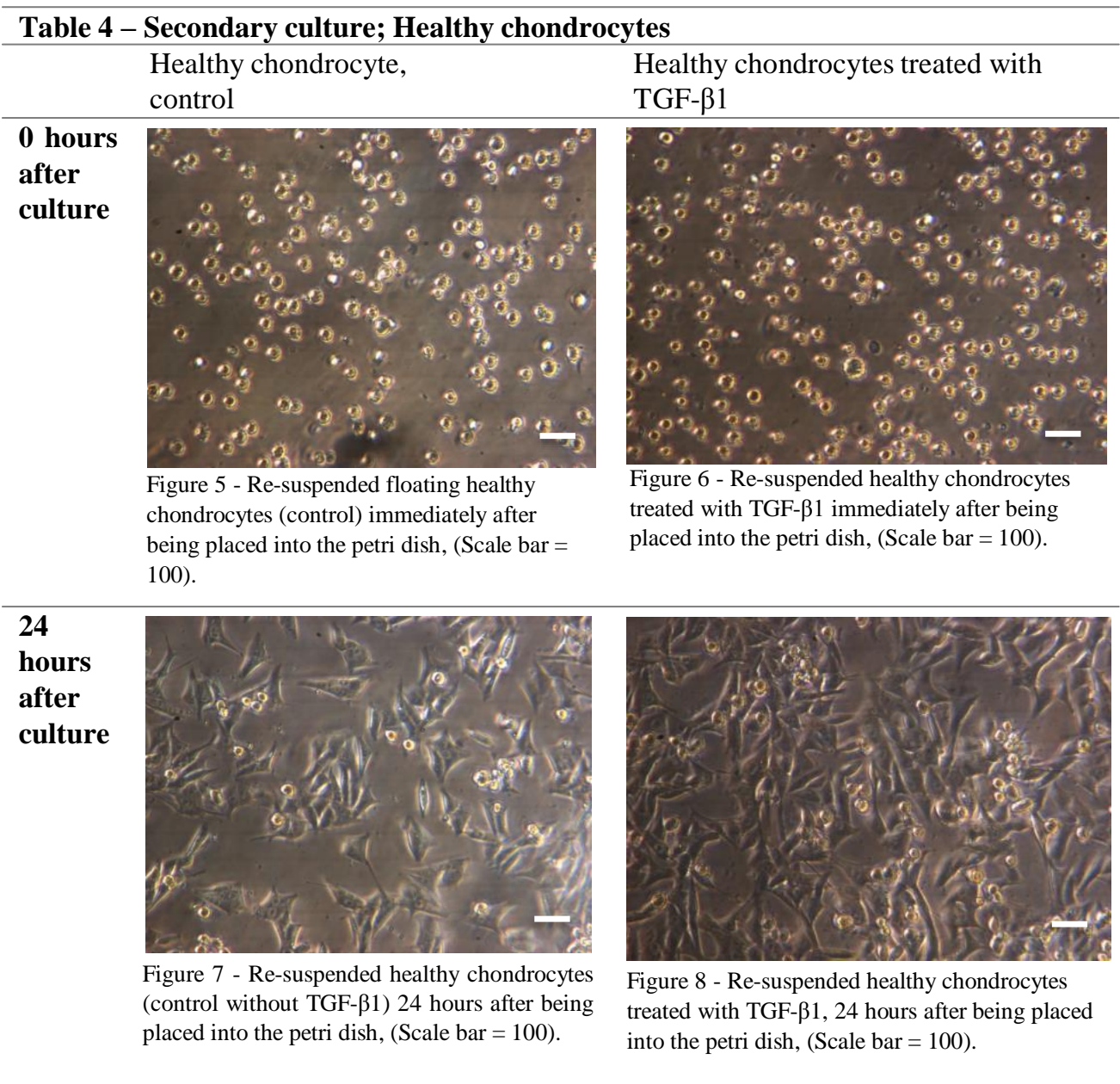

During cell isolation and culture a change in morphology was seen between healthy and osteoarthritic cells after the first subculture (See Table 2 and 3, Figures 1-4). The culture flask and petri dishes containing the osteoarthritic chondrocyte suspension after 24 hours contained media did not match the colour of the media in which the healthy chondrocytes are contained. The Culture of primary cells in the first 24 hours was successful, allowing acceptable doubling for a good quantity of cells to work with. Before the use of trypsin after 24 hours, fibroblast like attachments were observed; the orientation of the cells were randomised, which is a well-known sign for healthy chondrocytes in vitro [8].

The secondary culture of both healthy and degraded cells was also a success, providing us with microscopic images, which portrayed images of rounded morphology in healthy cartilage cells in vitro, alongside a visual aid of the more fibroblastic differentiated morphology of osteoarthritic cells in vitro (See Table 4, Figures 5-8). The difference in quantity could also be clearly seen, showing that in the same amount of time, healthy cells created a larger amount of cells compared to degraded cells.

The final forms of culture which has been treated with and without TGF- $\beta 1$ were also successful, portraying that the samples induced with TGF- $\beta 1$ (both healthy and osteoarthritic) contained a larger amount of Glycosaminoglycans, confirmed via quantification of cells after 24 hours of culture (See Table 5, Figures 9-12). Also the differentiation of 
morphology was observed after growth factor induction, from the rounded chondrocyte to more that of a fibroblastic change. The samples, which were cultured with standard DMEM, contained less viable cells, and the healthy cells did not have a dramatic change in terms of morphology.

\section{Table 5 - Secondary culture; Osteoarthritic chondrocytes}

Osteoarthritic chondrocyte (control)

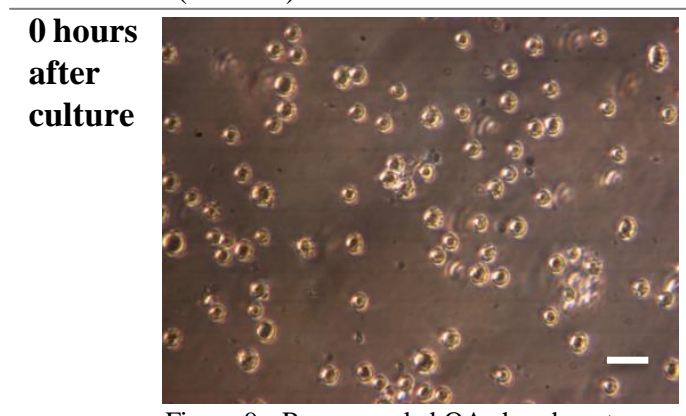

Figure 9 - Re-suspended OA chondrocytes (control without TGF- $\beta 1$ ) immediately after being placed into the petri dish, (Scale bar $=$ 100).
Osteoarthritic chondrocytes treated with TGF- $\beta 1$

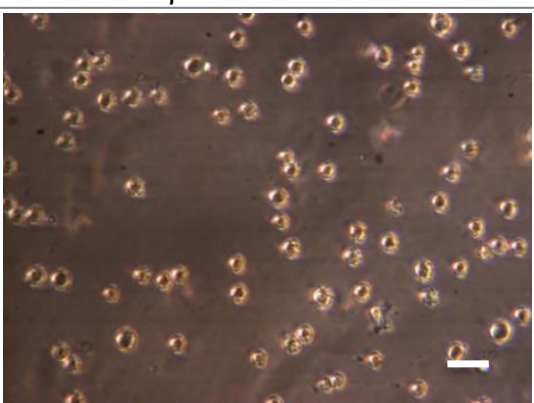

Figure 10 - Re-suspended OA chondrocytes treated and cultured with TGF- $\beta 1$ immediately after being placed into the petri dish, $($ Scale bar $=100)$.

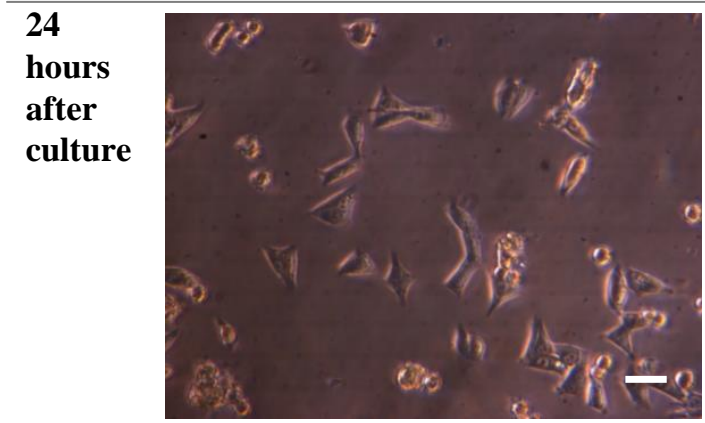

Figure 21 - Re-suspended OA chondrocytes (control, without TGF- $\beta 1$ ) 24 hours after being placed into the petri dish, $($ Scale bar $=100)$.

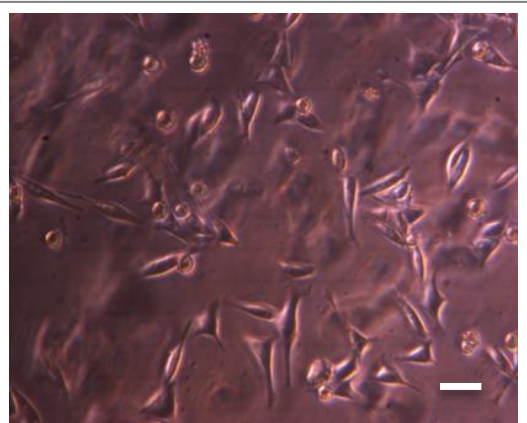

Figure 32 - Re-suspended OA chondrocytes treated and cultured with TGF- $\beta 1,24$ hours after being placed into the petri dish, (Scale bar = 100).

Analysis of glycosaminoglycan content using the SpectraMax $M 2 / M 2^{e}$ plate reader.

Figure 13 represent the glycosaminoglycan analysis and concentration curve for mean absorbance of DMMB dye in correlation to the concentration of exiting proteins via spectrophotometry analysis using the SoftPro max plate reader. This include " $\mathrm{HCH}-\mathrm{C}$ " (healthy chondrocytes control), "HCH + TGF $\beta 1$ " (healthy chondrocytes + TGF $\beta 1$ ), "OACH - C" (osteoarthritic chondrocyte control) and "OACH + TGF $\beta 1$ " (osteoarthritic chondrocyte + TGF $\beta 1$ ). 
A Concenctration curve to show the mean absorbance of $\mathrm{DMMB}$ dye in correlation to the concentration of protein present.

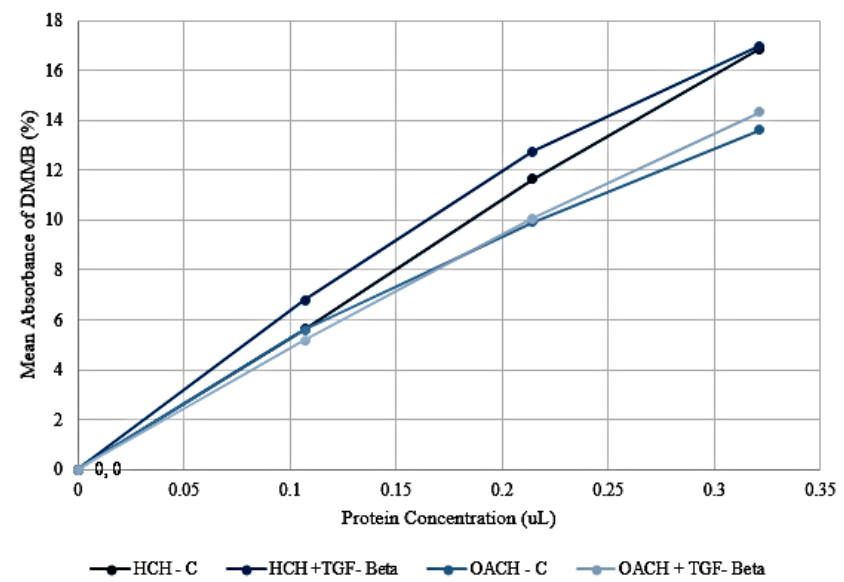

Figure 13 - Displays a protein concentration curve obtained from the values provided via spectrophotometry analysis using the SoftPro max plate reader. Legends include "HCH - C" (healthy chondrocytes control), "HCH + TGF $\beta 1$ " (healthy chondrocytes + TGF $\beta 1$ ), "OACH - C" (osteoarthritic chondrocyte control) and "OACH + TGF $\beta 1$ " (osteoarthritic chondrocyte + TGF $\beta 1$ ).

Samples treated with TGF- $\beta 1$ with more staining of GAGS with DMMB, shows that TGF- $\beta 1$ does most definitely retain water, and hence is a large factor to be considered for curing OA. Samples without treatment of TGF- $\beta 1$ showed less absorbance as a result of reduction in glycosaminoglycan synthesis. The concentration curves for GAG's in the output curve produced demonstrated higher absorbance in the samples containing TGF- $\beta 1$ than the samples without TGF- $\beta 1$ (See Figure 13). The osteoarthritic cells absorbed more light in plate reader compared with healthy chondrocytes, confirming that glycosaminoglycans are lost and less water is retained when osteoarthritis occurs. Also, absorbance values provided from the plate reader conveyed an increase in glycosaminoglycan staining in both the healthy and osteoarthritic samples induced with TGF- $\beta 1$ compared to their control groups, suggesting that the growth factor caused a slight form of upregulation in synthesis. Both variables when induced with $10 \mathrm{ng} / \mathrm{ml}$ of TGF $\beta 1$ increased absorption of DMMB; TGF- $\beta 1$ induced water retention in both healthy and osteoarthritic cells. 2 Sample T tests were carried out for all collected results.

The P-value was less than 0.05 for two proteins concentration; when the protein concentration was $32 \%$, the $\mathrm{P}$ - value was 0.004 and when the protein concentration was $21 \%$, the P-value was 0.011 . According to those values, one could assume GAG synthesis in healthy cells is significantly increased when compared to osteoarthritic cells and accept the alternative hypothesis.

\section{Conclusion}

The Culture of primary cells in the first 24 hours was successful, allowing acceptable doubling for a good quantity of cells to work with. The second culture of both the healthy and degraded cells was also a success, providing us with microscopic images, which portrayed images of rounded morphology in healthy cartilage cells in vitro, alongside a visual aid of the more fibroblastic differentiated morphology of osteoarthritic cells in vitro. Cells treated with and without TGF- $\beta 1$ were also successful, representing that the samples induced with TGF- $\beta 1$ (both healthy and osteoarthritic) contained a larger amount of Glycosaminoglycans. After treatment with TGF- $\beta 1$, rounded chondrocyte changed mainly to more fibroblastic morphology. The control samples did not show a dramatic change in terms of morphology. In conclusion, TGF- $\beta 1$ represent significant water retention for cartilage repair. 


\section{References}

[1] Morales TI, J. M. (1991). Transforming growth factor- $\beta$ in calf articular cartilage organ cultures: synthesis and distribution. Arch Biochem Biophys, 397-405

[2] Mariani, L. P. (2014, February 25). Signaling Pathways in Cartilage Repair. International Journal of Molecular Sciences, 8668,8669.

[3] Beggs, C.B., Denyer, M.C.T., Lemmerz, A., Sefat, F., Wright, C., Youseffi, M. (2010) "The effect of transforming growth factor beta (TGF- $\beta 3$ ) and Sanicle on wound healing", Proceedings of the World Congress on Engineering (WCE): International conference of systems biology and bioengineering (ICSBB), 2010, June 30th July 2nd, London, UK, Vol II, pp 572-577, (ISBN: 978-988-17012-9-9).

[4] Sefat, F. (2014) "Transforming Growth Factor Beta (TGF- $\beta$ ): Natural Curing Agents for Repair”, MOJ Cell Sci Report 1(3):00009.

[5] Sefat, F., Youseffi, M., Khaghani, S.A., Soon, C.F., Javid, F. (2016a) Effect of Transforming Growth Factor- $\beta 3$ on mono and multilayer chondrocytes, Cytokines, 83:118-26.

[6] Kaur, P., Khaghani, S.A., Khurshid, Z., Zafar, M.S., Mozafari, M., Sefat, F. (2017) Fabrication and Characterisations of Hydrogels for Cartilage Repair Advances in Tissue Engineering and Regenerative Medicine.

[7] Sefat, F., Youseffi, M., Berends, R.F., Khaghani, S.A., And Denyer, M.C.T. (2009) "The Effect of Transforming Growth Factor Beta (TGF- $\beta 3$ ), $\mathrm{HCl}$ and BSA/HCl on Trypsinisation of Bone Cells Monolayer," Proceedings of the World Congress on Engineering (WCE): International conference of systems biology and bioengineering (ICSBB), 2009, July 1 - 3, London, UK, Vol II, pp 1859-1862 (ISBN:978-988-18210-1-0).

[8] Khaghani, S. A. (2010). Cell and tissue engineering of articular cartilage via regulation and alignment of primary chondrocyte using manipulated transforming growth factors and ECM proteins. Bradford: University of Bradford.

[9] Khaghani, S.A., Sefat, F., Youseffi, M., Rehman, R., Soon, C.F., Akbarova, G. (2016) Effect of transforming growth factor- $\beta$ on up/down regulation of integrin- $\beta 1$ in primary chondrocyte culture. European Cells and Materials, TCES, August 2016, UCL, London, UK (ISSN 1473-2262).

[10] Sefat, F., Khaghani, S.A., Kaur, P., Agbabiaka, D., Youseffi, M. (2016b) Fabrication and Characterisation of Hydrogels for Cartilage Repair, Regenerative Medicine and Biomaterials Conference: MEIbioeng, 5-6 September 2016, Oxford, UK.

[11] Isabelle Barbosa, S. G.-C.-P.-G. (2003). Improved and simple micro assay for sulfated glycosaminoglycans quantification in biological extracts and its use in skin and muscle tissue studies. Glycobiology , --. 